\title{
Evaluation of Serum Tumor Markers Variation Following the Radioactive Iodine Therapy in Patients with Differentiated Thyroid Cancer
}

\author{
Sorush Niknamian* \\ Military Medicine Department, Liberty University, USA \\ *Corresponding author: Sorush Niknamian, Military Medicine Department, Liberty University, VA, USA
}

\begin{abstract}
Introduction: Radioactive iodine is the effective therapy in thyroid cancer. The aim of this study is to evaluate the serum tumor markers in patients under the therapy with radioactive iodine 131.

Material and Methods: 45 cases of female patients aged 16-60 years with thyroid cancer surgery referred to the nuclear medicine department of Nemazi hospital for (iodine treatment after surgery) were selected. The selection was on the basis of interviewing and information of patients is consent forms. Only patients with thyroid cancer and referred for the first time without any other diseases were chosen for this study. The selected patients were prescribed a dose of $150 \mathrm{mCi}$ of I-131. From each patient, $4 \mathrm{~mL}$ of chelated serum for serological studies on tumor markers and $2 \mathrm{~mL}$ of oxalated serum for spectrophotometry studies on cell death were used in three stages. The first stage before the iodine therapy, the second stage, after 48 hours, and the third stage, 30 days after radioiodine therapy were studied and the results were evaluated by the one-way repeated measures ANOVA test.

Results: according to the results of dependent paired T-Test, AFP, in the periods before, 48 hours and 1 month after radioiodine therapy, respectively were $3.46 \pm 1.21$ and $3.74 \pm 1.37$ and $3.76 \pm 1.25(\mathrm{p}<0.0005)$. About CA 19-9 in the periods before, 48 hours and one month after radioiodine therapy, the results were $9.30 \pm 6.32,9.95 \pm 6.92(\mathrm{p}=0.040)$ and $11.26 \pm 7.49(\mathrm{p}<0.0005)$ respectively. About CEA, the results were $1.60 \pm 0.60,1.47 \pm 0.55$ and $2.23 \pm 0.69(\mathrm{p}<0.0005)$, respectively. In the case of tumor marker CA 15-3 results were $15.53 \pm 6.48$ and $1.60 \pm 0.60$ and $15.68 \pm 6.52(\mathrm{p}=0.014)$, respectively and in the case of ALP, results were $124.22 \pm 5$ and $122.2 \pm 6$ and $116.7 \pm 7(\mathrm{p}<0.0005)$, respectively.

Conclusion: According to the same studies and the acquired results, it can be concluded that the tumor markers CEA and CA199 are more acceptable and sustainable for monitoring the malignancy and progressive disease in patients with thyroid cancer. The decreasing ALP is normal and transient. The increase of AFP and CA15-3 is not even statistically reliable. It is recommended that the period of iodine therapy and falsely elevated tumor markers can be informed to the doctor, during the gastrointestinal studies in patients with thyroid cancer, in order to prevent wrong decisions on the treatment process.
\end{abstract}

Keywords: Tumor marker; thyroid cancer; iodine therapy; iodine-131

\section{Introduction}

It is known for a long time that exposure to ionizing radiation is harmful to biological tissues and it can cause irreversible tissue damage, cancer and even death in the case of sufficient amounts and intensities [1]. Iodine therapy has been used for benign and malignant thyroid therapies since 1940 . When the radioactive iodine absorbed by the thyroid, the effect of the iodine therapy was started due to the high-energy beta radiation and it resulted in the cell death after a few weeks to a few months [2]. The incidence rates of thyroid cancer in both women and men have been increasing in recent years. This year, an estimated 64,300 adults (14,950 men and 49,350 women) in the United States will be diagnosed with thyroid cancer. Thyroid cancer is the fifth most common cancer in 
women. Women are 3 times more likely to have thyroid cancer than men, but women and men die at equal rates. This suggests that men have a worse prognosis than women when there is a diagnosis of thyroid cancer [3]. Most types of thyroid cancer are papillary and follicular thyroid cancer which can be surgically removed and since their forming cells absorb iodine as normal cells do, the residual cells can be destroyed with radioactive iodine after surgery. Beta particles of iodine can destroy follicular cells and gradually leads to mass reduction and thyrotoxicosis.

As a result, iodine therapy will perform to destroy remaining thyroid tissue and prevent the metastatic potential. The major feature of thyroid cancer cells that differentiate it from the rest of cancers is to absorb iodine strongly. As a result, iodine concentrates in the remaining thyroid cells destroys them and the other cells that do not absorb iodine will remain safe [4]. Thyroid cancer therapy methods include surgery, radiotherapy, and chemotherapy. Radiation therapy performs thorough either radioactive iodine or external radiation therapy procedures. After thyroidectomy for thyroid cancer treatment, it should be ensured that no cancer cells are left. Despite many types of cancer, chemotherapy is not an effective treatment for metastatic thyroid cancer. These patients would be treated with radioactive iodine. Iodine therapy can help to maximize the therapeutic effect in the next stages of treatment procedure and it also reduces recurrence, mortality and morbidity and prevents the metastasis to other targets [5]. Radioactive iodine is often used in imaging technology, hyperthyroidism treatment, thyroid cancer and other types of cancer. In radiography studies such as thyroid scan, the patient receives a small dose of radioactive iodine that accumulates in thyroid cells or in certain types of tumors and it can be detected using a scanner. More concentrated iodine areas represent the abnormal activity of thyroid cells (hot thyroid nodules). Radioactive iodine is used in internal radiation therapy for prostate cancer, intraocular melanoma and carcinoid tumors (brachytherapy). In order to destroy cancer cells, the radioactive iodine, in liquid form or oral capsules is applied into or near the tumor via infusion or implantation [6]. A tumor marker is a chemical produced by the tumor itself or by the normal cells responding to a tumor. Some tumor markers are completely specific for particular cancer such as PSA for prostate while others, such as CIA increases in various cancers, including colon, stomach, liver, pancreas, lung and breast, and they are nonspecific [7]. From the clinical point of view, an ideal tumor marker not only should be perfectly specific for particular cancer but also it should be quite sensitive and accurate for early detection of small tumors. Unfortunately, only a few numbers of specific tumor markers are specific, and the others are not specific and most of them are found in many tumors [8].

From the structural and biochemical point of view, tumor markers are in a large variety. Sometimes a simple analytic such as ferritin, which previously only assessed as a protein, is now categorized as a tumor marker and sometimes complex compounds such as estrogen and progesterone receptors that are associated with breast cancer are considered as tumor markers [9-11]. High levels of ionizing radiation from radioactive substances are harmful to organisms, including humans. Ionizing radiation (alpha, beta, gamma and neutron) with enough energy can damage the molecular structure and thereby cause cell dysfunction and mutation. Beam radiation may cause adverse effects on the hematopoietic system, gastrointestinal tract, central nervous system and ultimately affect the whole body (Figures 1-5). The level of sensitivity of the cells is different for example, hematopoietic cells are the most sensitive cells to radiation [12]. Ionizing radiation creates excited and ionized atoms and molecules. The excitation can generate free radicals, breaks the chemical bonds and connectivity between macromolecules and produces new chemical bonds and finally damages to the regulator molecules of living cells [13].

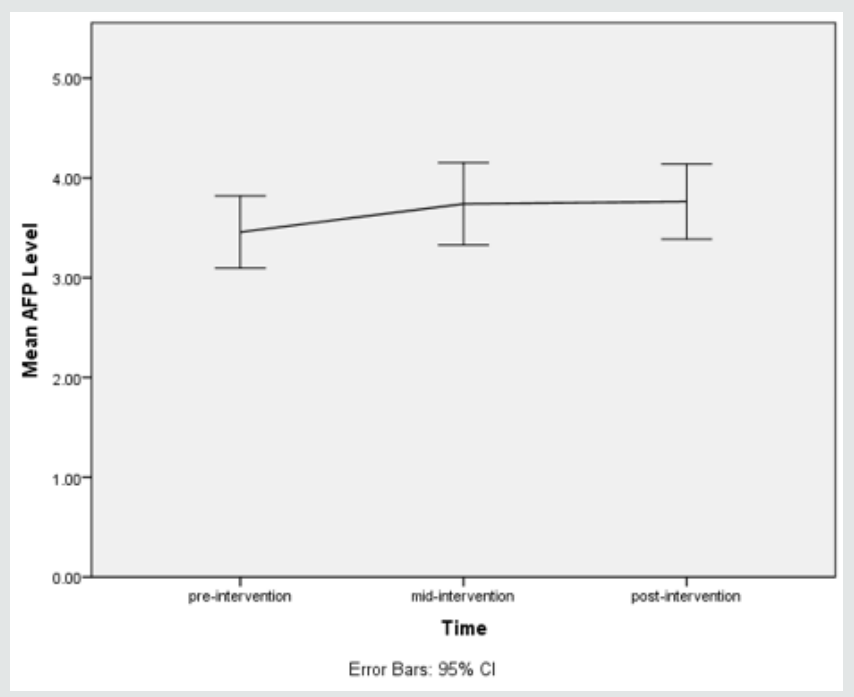

Figure 1. 


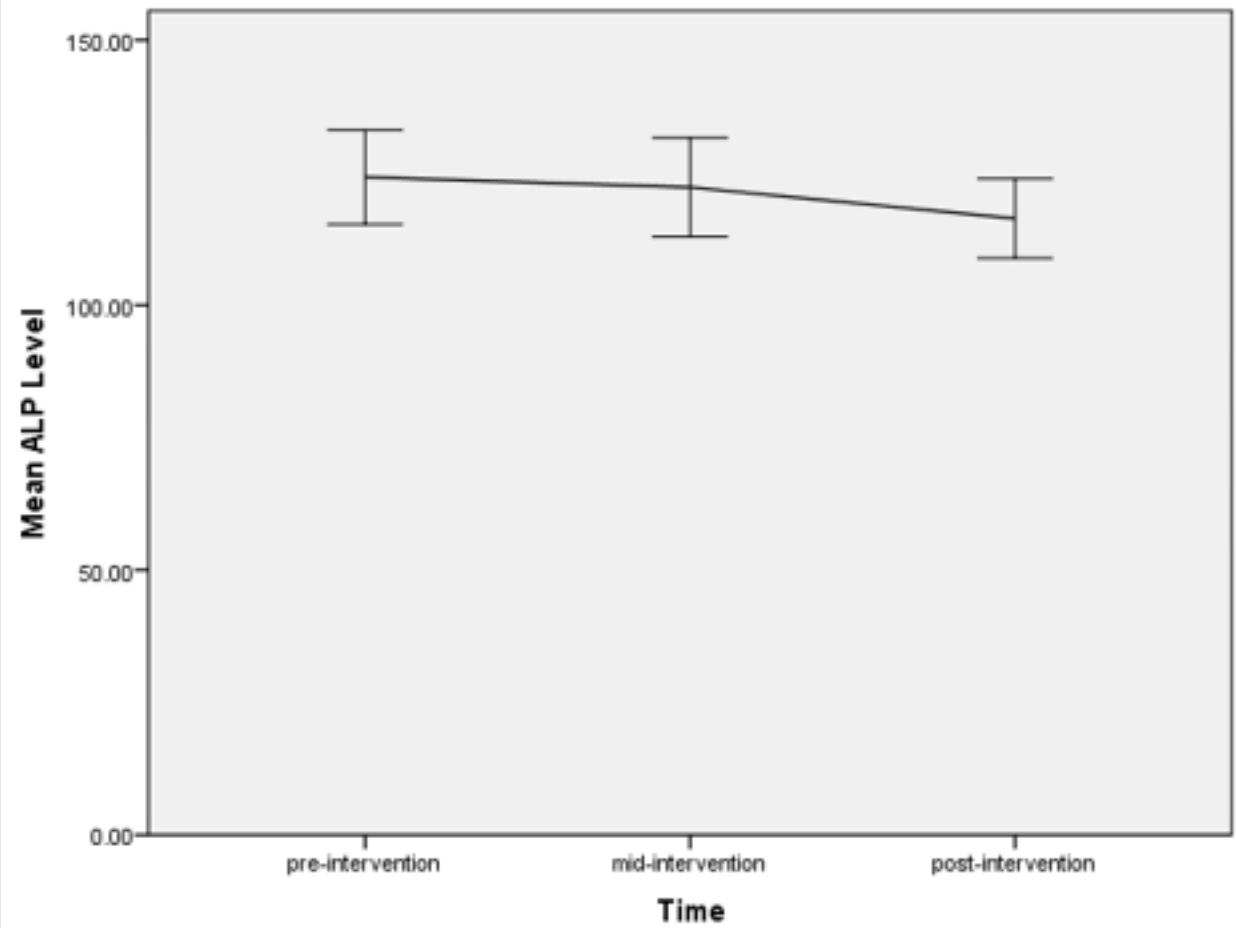

Error Bars: $95 \% \mathrm{Cl}$

Figure 2.

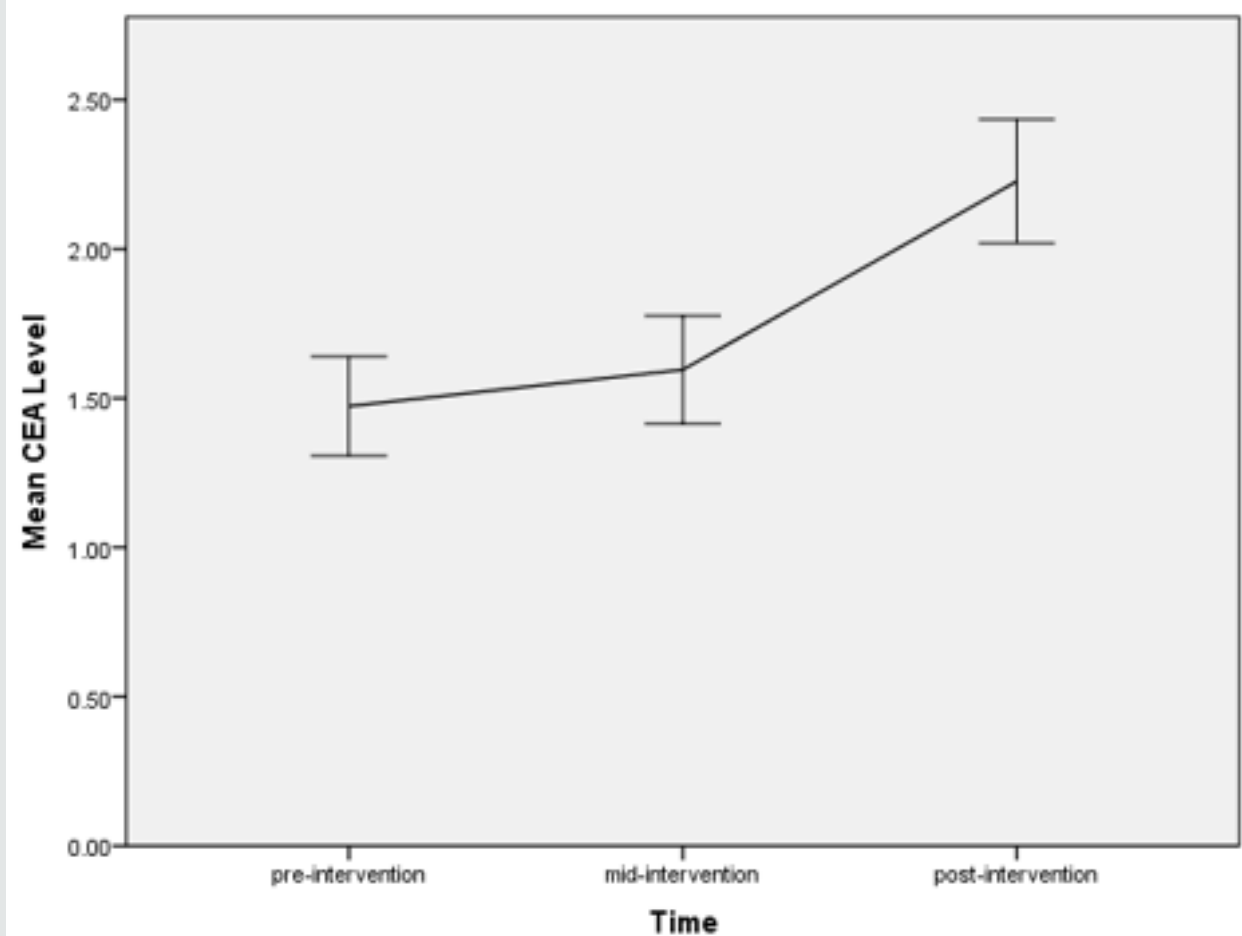

Error Bars: $95 \% \mathrm{Cl}$

Figure 3. 


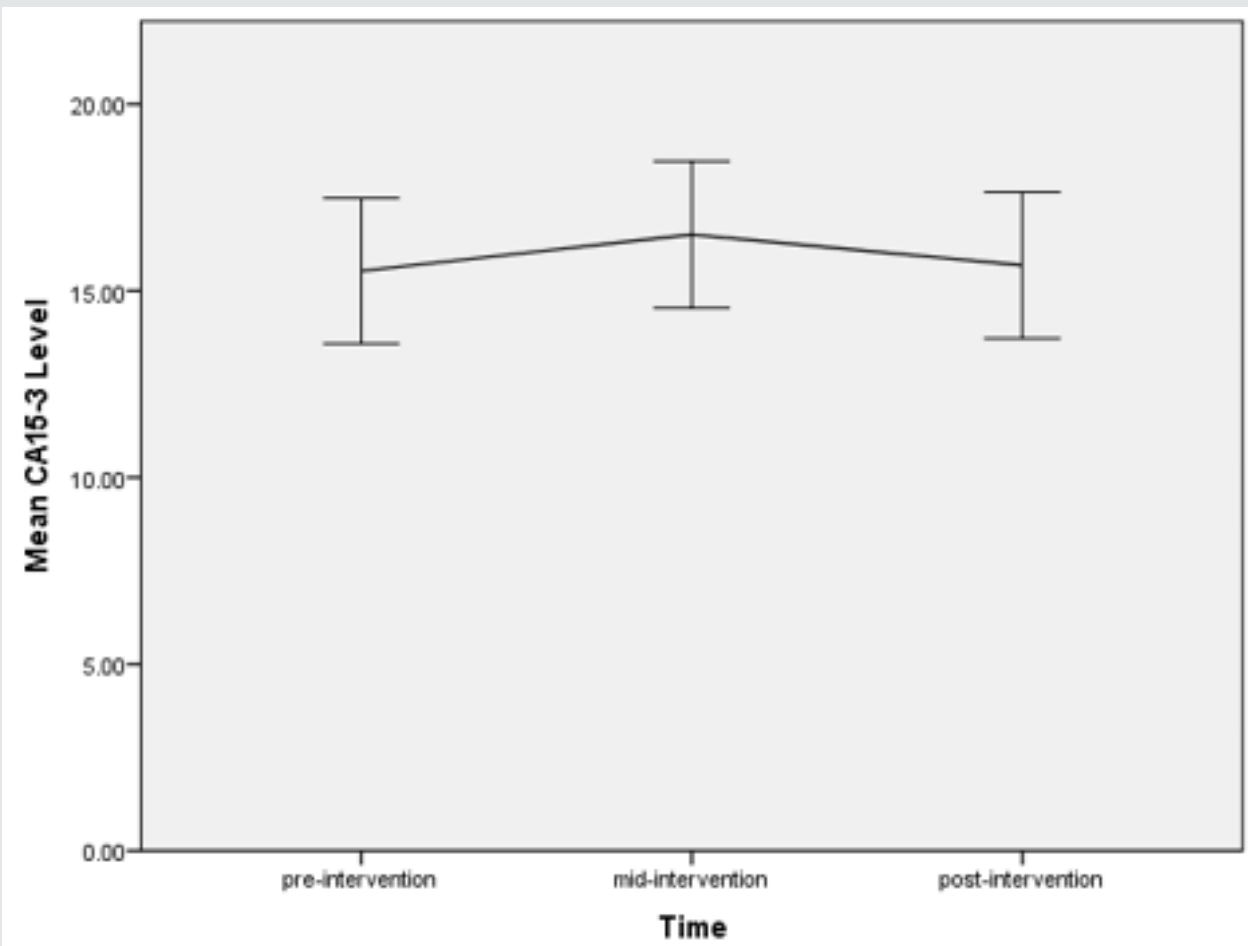

Error Bars: $95 \% \mathrm{Cl}$

Figure 4.

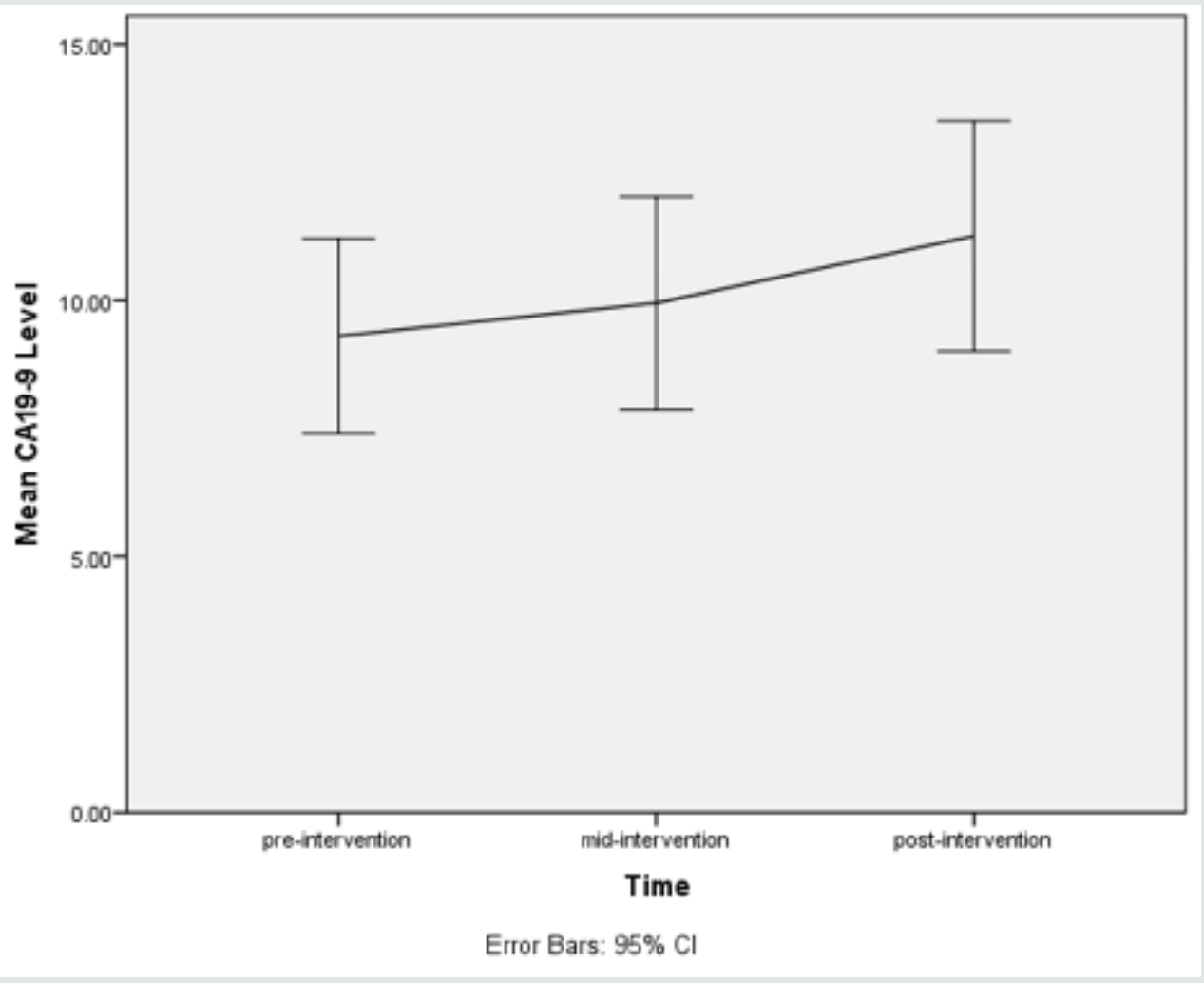

Figure 5. 


\section{Material and Methods}

This study is a cross-sectional, descriptive, and analytical study. In general, the stages of this research can be categorized as follows: The first stage: Identifying the patients and obtain informed consent from them; The second stage: Blood samples were taken from patients; The third stage: Related experiments were done. 45 cases of female patients aged 16-60 years with thyroid cancer surgery referred to the nuclear medicine department of Nemazi hospital for treatment (iodine treatment after surgery) were selected. The selection was on the basis of interviewing and information of patients is consent forms. Only patients with thyroid cancer and referred for the first time without any other diseases were chosen for this study. The selected patients were prescribed a dose of $150 \mathrm{mCi}$ of radioiodine therapy. From each patient, $4 \mathrm{~mL}$ of chelated serum for serological studies on tumor markers and 2 $\mathrm{mL}$ of oxalate serum for spectrophotometry studies on cell death were taken in three stages. The first stage before the iodine therapy, the second stage, after 48 hours, and the third stage, 30 days after radioiodine therapy were studied. Blood samples were obtained and after separation of serum, the level of serum tumor markers including AFP, ALP, CEA, CA15-3, and CA19-9 were analyzed by chemiluminescence method (Architect Instrument).

\section{Statistical analysis}

The gathered data were analyzed using SPSS V.20 software. The one-way repeated measures ANOVA test was used to conclude whether there was a statistically significant difference in the level of measured tumor markers in patients' serum due to I-131 therapy. All tumor markers serum levels were expressed as mean \pm SD and $p$ values less than 0.05 were considered statistically significant.

\section{Results}

\section{a. Results of measured levels of AFP}

A one-way repeated measures ANOVA was conducted to determine whether there was a statistically significant difference in AFP concentration as a result of radioiodine therapy. There were no outliers, and the data was normally distributed at each time point, as assessed by boxplot and Shapiro-Wilk test ( $p>$ $0.05)$, respectively. The assumption of sphericity was met, as assessed by Mauchly's test of sphericity, $\chi 2(2)=0.425, p=0.809$. Radioiodine therapy elicited statistically significant changes in AFP concentration over time, $F(2,88)=10.528, p<0.0005$, partial $\eta 2$ $=0.193$, with AFP concentration increasing from $3.46 \pm 1.21 \mathrm{ng} /$ $\mathrm{mL}$ pre-intervention to $3.74 \pm 1.37 \mathrm{ng} / \mathrm{mL}$ at 48 hours and to 3.76 $\pm 1.25 \mathrm{ng} / \mathrm{mL}$ at 1 month (post-intervention). Post hoc analysis with a Bonferroni adjustment revealed that AFP concentration was statistically significantly increased from pre-intervention to 48 hours ( 0.28 ( $95 \% \mathrm{CI}, 0.11$ to 0.46$) \mathrm{ng} / \mathrm{mL}, \mathrm{p}=0.001$ ), and from preintervention to post-intervention $(0.30$ (95\% CI, 0.11 to 0.49$) \mathrm{ng} /$
$\mathrm{mL}, \mathrm{p}=0.001)$, but not from 48 hours to post-intervention $(0.02$ ( $95 \% \mathrm{CI},-0.16$ to 0.21$) \mathrm{ng} / \mathrm{mL}, \mathrm{p}=1$ ).

\section{b. Results of measured levels of ALP}

A one-way repeated measures ANOVA was conducted to determine whether there was a statistically significant difference in ALP concentration as a result of radioiodine therapy. There were no outliers, and the data was normally distributed at each time point, as assessed by boxplot and Shapiro-Wilk test ( $\mathrm{p}>$ $0.05)$, respectively. The assumption of sphericity was violated, as assessed by Mauchly's test of sphericity, $\chi 2(2)=16.113, p<$ 0.0005 . Therefore, a Greenhouse-Geisser correction was applied ( $\varepsilon=0.762$ ). Radioiodine therapy elicited statistically significant changes in ALP concentration over time, $\mathrm{F}(1.524,67.046)=13.027$, $\mathrm{p}<0.0005$, partial $\eta 2=0.228$, with ALP concentration decreasing from $124.13 \pm 29.63 \mathrm{U} / \mathrm{L}$ pre-intervention to $122.22 \pm 31.01 \mathrm{U} / \mathrm{L}$ at 48 hours and to $116.33 \pm 24.92 \mathrm{U} / \mathrm{L}$ at 1 month (post-intervention). Post hoc analysis with a Bonferroni adjustment revealed that ALP concentration was statistically significantly decreased from preintervention to post-intervention (7.8 (95\% CI, 5.09 to 10.60$) \mathrm{U} / \mathrm{L}$, $\mathrm{p}<0.0005$ ), and from 48 hours to post-intervention (5.89 (95\% CI, 1.18 to 0.49 ) $\mathrm{U} / \mathrm{L}, \mathrm{p}=0.001$ ), but not from pre-intervention to 48 hours (1.91 (95\% CI, 2.29 to 6.11) U/L, p = 0.79).

\section{c. Results of measured levels of CEA}

A one-way repeated measures ANOVA was conducted to determine whether there was a statistically significant difference in CEA level as a result of radioiodine therapy. There were no outliers, and the data was normally distributed at each time point, as assessed by boxplot and Shapiro-Wilk test ( $p>0.05$ ), respectively. The assumption of sphericity was violated, as assessed by Mauchly's test of sphericity, $\chi 2(2)=26.328, p<0.0005$. Therefore, a Greenhouse-Geisser correction was applied $(\varepsilon=0.686)$. Radioiodine therapy elicited statistically significant changes in CEA level over time, $\mathrm{F}(1.372,60.361)=146.821, \mathrm{p}<0.0005$, partial $\eta 2=0.769$, with CEA level increasing from $1.47 \pm 0.55 \mathrm{ng} / \mathrm{mL}$ pre-intervention to $1.60 \pm 0.60 \mathrm{ng} / \mathrm{mL}$ at 48 hours and to $2.23 \pm 0.69 \mathrm{ng} / \mathrm{mL}$ at 1 month (post-intervention). Post hoc analysis with a Bonferroni adjustment revealed that CEA level was statistically significantly increased from pre-intervention to mid-intervention 0.12 (95\% CI, 0.05 to 0.19 ) $\mathrm{ng} / \mathrm{mL}, \mathrm{p}<0.0005$ ), and from pre-intervention to post-intervention ( 0.75 ( $95 \% \mathrm{CI}, 0.61$ to 0.90$) \mathrm{ng} / \mathrm{mL}, \mathrm{p}<0.0005$ ), and from mid-intervention to post-intervention $(0.63$ (95\% CI, 0.50 to 0.76$) \mathrm{ng} / \mathrm{mL}, \mathrm{p}<0.0005$ ).

\section{d. Results of measured levels of CA15-3}

A one-way repeated measures ANOVA was conducted to determine whether there was a statistically significant difference in CA15-3 level as a result of radioiodine therapy. There were no outliers, and the data was normally distributed at each time point, as assessed by boxplot and Shapiro-Wilk test (p > 
0.05), respectively. The assumption of sphericity was violated, as assessed by Mauchly's test of sphericity, $\chi 2(2)=65.611, p<$ 0.0005 . Therefore, a Greenhouse-Geisser correction was applied ( $\varepsilon=0.561$ ). Radioiodine therapy elicited statistically significant changes in CA15-3 level over time, $F(1.122,49.367)=6.950, p=$ 0.009 , partial $\eta 2=0.136$, with CA15-3 level increasing from 15.53 $\pm 6.48 \mathrm{U} / \mathrm{mL}$ pre-intervention to $16.50 \pm 6.53 \mathrm{U} / \mathrm{mL}$ at 48 hours and to $15.68 \pm 6.52 \mathrm{U} / \mathrm{mL}$ at 1 month (post-intervention). Post hoc analysis with a Bonferroni adjustment revealed that CA15-3 level was statistically significantly increased from pre-intervention to mid-intervention (0.97 ( $95 \% \mathrm{CI}, 0.16$ to 1.78 ) U/mL, p = 0.01), but not from pre-intervention to post-intervention ( 0.15 (95\% CI, - 0.09 to 0.39$) \mathrm{U} / \mathrm{mL}, \mathrm{p}=0.39$ ). There was a reduction in CA15-3 level from mid-intervention to post-intervention $(0.82$ (95\% CI, -1.69 to $0.04) \mathrm{U} / \mathrm{mL}, \mathrm{p}=0.07$ ).

\section{e. Results of measured levels of CA19-9}

A one-way repeated measures ANOVA was conducted to determine whether there was a statistically significant difference in CA19-9 level as a result of radioiodine therapy. There were no outliers, and the data was normally distributed at each time point, as assessed by boxplot and Shapiro-Wilk test ( $\mathrm{p}>$ $0.05)$, respectively. The assumption of sphericity was violated, as assessed by Mauchly's test of sphericity, $\chi 2(2)=20.603, p<$ 0.0005 . Therefore, a Greenhouse-Geisser correction was applied ( $\varepsilon=0.724$ ). Radioiodine therapy elicited statistically significant changes in CA19-9 level over time, $\mathrm{F}(1.449,63.736)=6.950, \mathrm{p}<$ 0.0005 , partial $\eta 2=0.420$, with CA19-9 level increasing from 9.30 $\pm 6.32 \mathrm{U} / \mathrm{mL}$ pre-intervention to $9.94 \pm 6.92 \mathrm{U} / \mathrm{mL}$ at 48 hours and to $11.25 \pm 7.49 \mathrm{U} / \mathrm{mL}$ at 1 month (post-intervention). Post hoc analysis with a Bonferroni adjustment revealed that CA19-9 level was statistically significantly increased from pre-intervention to mid-intervention $(0.65$ ( $95 \% \mathrm{CI}, 0.02$ to 1.27$) \mathrm{U} / \mathrm{mL}, \mathrm{p}=0.04)$, and from pre-intervention to post-intervention (1.95 (95\% CI, 1.18 to 2.72$) \mathrm{U} / \mathrm{mL}, \mathrm{p}<0.0005)$, and from mid-intervention to postintervention (1.31 (95\% CI, 0.89 to 1.73 ) $\mathrm{U} / \mathrm{mL}, \mathrm{p}<0.0005)$.

\section{Discussion}

In medical applications of Ionizing radiation, such as radiology, nuclear medicine and CT scan, the risk of exposure should be considered in addition to the advantages such as diagnosis and treatment of diseases. Radioactive iodine 131 is a radioactive material that has many applications in nuclear medicine such as diagnosis and treatment so that its doses used for diagnosis is about $5 \mathrm{mCi}$. However, the doses used for treatment is much higher near 20 to $250 \mathrm{mCi}$ and even higher [14]. This substance is used at higher doses than $100 \mathrm{mCi}$ for the treatment of thyroid cancers and at lower doses for treatment of hyperthyroidism. One of the major differences between radioactive iodine 131 with other radio-drugs that are used in nuclear medicine is the type of emitted radiation that is beta, and it has much more biological effects than gammaemitter [14]. In a study that was conducted in 1997 in Hungary, the sensitivity of the tumor marker CA19-9 in cancers of the stomach, intestines and pancreas were examined and the results are absolutely in favor of pancreatic cancer [15]. In a study conducted in the Czech Republic by Vrbikova et al. on a number of anaplastic thyroid cancer patients, high levels of this tumor marker was observed and after detailed imaging studies, they concluded that the results are false-positive and not reliable in a long time [16]. In our patients, at various periods, a significant increase in CA19-9 levels is detected at 48 hours after receiving I 131 compared to preinjection $(\mathrm{p}=0.040)$.

After one month, it can be seen that CA19-9 level was increasing in patients. This increase compared to pre-receiving I131 and 48 hours after its receipt is quite significant and confirms the results of previous study. This can cause a problem in the study of gastrointestinal diseases and lead to a false positive. Raised AFP alludes to a state where AFP levels are outside of the reference run. There are two classifications of AFP tests: tests performed on serum (blood plasma), and tests performed on amniotic fluid. Tests performed on serum are additionally classified by the purpose behind playing out the test: maternal serum, grown-up tumor marker, and pediatric tumor marker. Abnormally elevated AFP in the serum of a pregnant woman can have one or more of these sources: a problem with the fetus; a problem with the placenta; and a tumor or liver disease in the woman [17]. Foremost tumors that discharge AFP are endodermal sinus tumor (yolk sac carcinoma), neuroblastoma, hepatoblastoma, and hepatocellular carcinoma. In patients with AFP-discharging tumors, serum levels of AFP frequently associate with tumor estimate. Resection is normally connected with a fall in serum levels. Serum levels are helpful in evaluating reaction to treatment (Table1-5) [18]. Like any raised tumor marker, elevated AFP without anyone else is not symptomatic, just suggestive. Tumor markers are utilized basically to screen the after effect of a treatment (e.g. chemotherapy). On the off chance that levels of AFP go down after treatment, the tumor is not developing. On account of infants, after treatment, AFP ought to go down quicker than it would typically.

Table 1.

\begin{tabular}{|c|c|c|c|c|c|c|}
\hline Time & Mean & SD & Pairwise Comparisons & Mean Difference & S.E. & p \\
\hline pre-intervention & 3.46 & 1.21 & 48 hours/pre-intervention & 0.28 & 0.07 & 0.001 \\
\hline mid-intervention (48 hrs) & 3.7 & 1.37 & post-intervention/pre-intervention & 0.3 & 0.077 & 0.001 \\
\hline post-intervention & 3.76 & 1.25 & post-intervention/48 hours & 0.02 & 0.075 & 1 \\
\hline
\end{tabular}


Table 2.

\begin{tabular}{|c|c|c|c|c|c|c|}
\hline Time & Mean & SD & Pairwise Comparisons & Mean Difference & S.E. & p \\
\hline pre-intervention & 124.13 & 29.63 & 48 hours/pre-intervention & 1.91 & 1.69 & 0.79 \\
\hline mid-intervention (48 hrs) & 122.22 & 31.01 & post-intervention/pre-intervention & 7.8 & 1.09 & $<0.0005$ \\
\hline post-intervention & 116.33 & 24.92 & post-intervention/48 hours & 5.89 & 1.89 & 0.001 \\
\hline
\end{tabular}

Table 3.

\begin{tabular}{|c|c|c|c|c|c|c|}
\hline Time & Mean & SD & Pairwise Comparisons & Mean Difference & S.E. & p \\
\hline pre-intervention & 3.46 & 1.21 & 48 hours/pre-intervention & 0.12 & 0.03 & $<0.0005$ \\
\hline mid-intervention (48 hrs) & 3.7 & 1.37 & post-intervention/pre-intervention & 0.75 & 0.06 & $<0.0005$ \\
\hline post-intervention & 3.76 & 1.25 & post-intervention/48 hours & 0.63 & 0.05 & $<0.0005$ \\
\hline
\end{tabular}

Table 4.

\begin{tabular}{|c|c|c|c|c|c|c|}
\hline Time & Mean & SD & Pairwise Comparisons & Mean Difference & S.E. & $\mathbf{p}$ \\
\hline pre-intervention & 15.53 & 6.48 & 48 hours/pre-intervention & 0.97 & 0.33 & 0.01 \\
\hline mid-intervention (48 hrs) & 16.5 & 6.53 & post-intervention/pre-intervention & 0.1 & 0.06 & 0.39 \\
\hline post-intervention & 15.68 & 6.52 & post-intervention/48 hours & 0.35 & 0.05 & 0.07 \\
\hline
\end{tabular}

Table 5.

\begin{tabular}{|c|c|c|c|c|c|c|}
\hline Time & Mean & SD & Pairwise Comparisons & Mean Difference & S.E. & $\mathbf{p}$ \\
\hline pre-intervention & 9.3 & 6.32 & 48 hours/pre-intervention & 0.65 & 0.25 & 0.04 \\
\hline mid-intervention (48 hrs) & 9.94 & 6.92 & post-intervention/pre-intervention & 1.95 & 0.31 & $<0.05$ \\
\hline post-intervention & 11.25 & 7.49 & post-intervention/48 hours & 1.31 & 0.17 & $<0.05$ \\
\hline
\end{tabular}

A transitory increment in AFP instantly taking after chemotherapy may show not that the tumor is developing yet rather that it is contracting [19]. In this study, we show changing in AFP after Radioiodine therapy. If a woman is pregnant after radioiodine therapy, it is unclear to recognize the main reason of AFP increased. According to radioactive iodine uptake in the liver, there is the anticipation of increasing of AFP tumor marker. As reported in this study, the level of this tumor marker in the serum was increased slightly and some of this increase was related to the change of hepatocytes. Remarkably, no study has been done in this case. In this study, the CEA level has increased too, especially in patients with metastasis report of their whole-body scan. There are many studies about changes in levels of CEA tumor marker, particularly in patients with thyroid cancer that they all have confirmed the increase of this tumor marker in patients with thyroid cancer [20-22]. CEA is a tumor marker, especially for cancers of the gastrointestinal tract. When the CEA level is abnormally high before surgery or other treatment, it is expected to fall to normal following successful surgery to remove all of cancer. A rising CEA level indicates progression or recurrence of cancer [23,24]. In our study, CEA increased after radioiodine therapy. The same as AFP It is unclear rising CEA-related to gastrointestinal diseases or a false positive about radioiodine therapy complications. In Hashimoto et al. study on changes in CA15-3 tumor marker in 111 patients with papillary cancer, they detected increased levels of this tumor marker in the blood after iodine therapy. As they reported, this increase was not defensible in comparison with a marker such as TG [25]. According to the results of this study, this tumor marker is not suitable for the evaluation of thyroid cancer stages. According to the iodine absorption in the gastrointestinal tract and liver, ALP level increases and decreases and false positive or false negative is the only scientific explanation. According to the same studies and the results, it can be concluded that CEA and CA19-9 tumor markers are more acceptable and sustainable for monitoring of malignancy and wider dimensions of disease in patients with thyroid cancer. Increase or decrease of ALP is natural and transient and increase in AFP and CA15-3 are not even reliable statistically. It is recommended that in the gastrointestinal studies of patients with thyroid cancer, the time periods of iodine therapy and the time interval that tumor markers can be falsely elevated, should be informed to the doctor in order to avoid making wrong decisions. It is also suggested for future studies to evaluate tumor marker in a longer period of time.

\section{Conclusion}

According to the same studies and the acquired results, it can be concluded that the tumor markers CEA and CA19-9 are more acceptable and sustainable for monitoring the malignancy and progressive disease in patients with thyroid cancer. The decreasing ALP is normal and transient. The increase of AFP and CA15-3 is not even statistically reliable. It is recommended that the period of iodine therapy and falsely elevated tumor markers can be informed 
to the doctor, during the gastrointestinal studies in patients with thyroid cancer, in order to prevent wrong decisions on the treatment process.

\section{Reference}

1. Li LB, Wang JP, Yu XR, He SS, Yu FH (2001) Medical Radiation Usage and Exposures from Medical X Ray Diagnosis in Shandong Province of China. Radiation Protection Dosimetry 93(3): 261-266.

2. Creach KM, Siegel BA, Nussenbaum B, Grigsby PW (2012) Radioactive Iodine Therapy Decreases Recurrence in Thyroid Papillary Microcarcinoma. ISRN Endocrinology pp. 816386.

3. Siegel R, Naishadham D, Jemal A (2012) Cancer statistics, 2012. CA: A cancer journal for clinicians 62(1): 10-29.

4. La Perle KM, Kim DC, Hall NC, Bobbey A, Shen DH, et al. (2013) Modulation of sodium/iodide symporter expression in the salivary gland. Thyroid Official Journal of the American Thyroid Association 23(8): 1029-1036.

5. Petrich T, Widjaja A, Musholt TJ, Hofmann M, Brunkhorst T, et al. (2001) Outcome after radioiodine therapy in 107 patients with differentiated thyroid carcinoma and initial bone metastases: side-effects and influence of age. European journal of nuclear medicine 28(2): 203-208.

6. Schrohl AS, Holten-Andersen M, Sweep F, Schmitt M, Harbeck N, et al. (2003) Tumor markers: from laboratory to clinical utility. Molecular \& cellular proteomics MCP 2(6): 378-387.

7. Diamandis EP, van der Merwe DE (2005) Plasma protein profiling by mass spectrometry for cancer diagnosis: opportunities and limitations. Clinical cancer researches an Official journal of the American Association for Cancer Research 11(3): 963-965.

8. Wagner PL, Austin F, Sathaiah M, Magge D, Maduekwe U, et al. (2013) Significance of Serum Tumor Marker Levels in Peritoneal Carcinomatosis of Appendiceal Origin. Annals of surgical oncology 20(2): 506-514.

9. Duffy MJ (2013) Tumor markers in clinical practice: a review focusing on common solid cancers. Medical principles and practice : international journal of the Kuwait University Health Science Centre 22(1): 4-11.

10. Duffy MJ (2007) Role of tumor markers in patients with solid cancers: A critical review. European journal of internal medicine 18(3): 175-184.

11. Carmignani CP, Hampton R, Sugarbaker CE, Chang D, Sugarbaker PH (2004) Utility of CEA and CA 19-9 tumor markers in diagnosis and prognostic assessment of mucinous epithelial cancers of the appendix. Journal of Surgical Oncology 87(4): 162-166.

12. Mortazavi SMJ, Ikushima T, Sharafi AA (1999) Radiation Hormesis and Adaptive Responses Induced by Low Doses of Ionizing Radiation. Journal of Kerman University of Medical Sciences 6(1): 50-60.

13. Tavakoli M R, Moradalizadeh M, Ananisarab Gh R, Hosseini Sm (2012) Evaluation of Blood Cell Count in The Radiology Staff Of Birjand Hospitals In 2011. Modern Care Journal 9(2): 80-86.
14. Watanabe N, Yokoyama K, Kinuya S, Shuke N, Shimizu M, et al. (1998) Radiotoxicity after iodine-131 therapy for thyroid cancer using the micronucleus assay. Journal of nuclear medicine official publication Society of Nuclear Medicine 39(3): 436-440.

15. Czako L, Takacs T, Babarczy E, Dux L, Lonovics J (1997) Comparative study of the role of CA 19-9, CA 72-4 and CEA tumor antigens in the diagnosis of pancreatic cancer and other gastrointestinal malignant diseases. Orvosi hetilap138(47): 2981-2985.

16. Vrbikova J, Trubac M, Adamek S, Zeman V, Duskova2 J (2010) An increase in serum CA 19-9 leading to the discovery of thyroid cancer in patient with the history of rectosigmoid cancer. Endocrine Abstracts 22: 762.

17. Bredaki FE, Wright D, Akolekar R, Cruz G, Nicolaides KH (2011) Maternal serum alpha-fetoprotein in normal pregnancy at 11-13 weeks' gestation. Fetal diagnosis and therapy 30(4): 274-279.

18. Duffy MJ (2004) Evidence for the clinical use of tumour markers. Annals of clinical biochemistry 41(Pt 5): 370-377.

19. Crocoli A, Madafferi S, Jenkner A, Zaccara A, Inserra A (2008) Elevated serum alpha-fetoprotein in Wilms tumor may follow the same pattern of other fetal neoplasms after treatment: evidence from three cases. Pediatric surgery international 24(4): 499-502.

20. Barbet J, Campion L, Kraeber-Bodere F, Chatal JF (2005) Prognostic impact of serum calcitonin and carcinoembryonic antigen doublingtimes in patients with medullary thyroid carcinoma. The Journal of clinical endocrinology and metabolism 90(11): 6077-6084.

21. Osamura RY, Yasuda O, Kawakami T, Itoh Y, Inada K, et al. (1997) Immunoelectron microscopic demonstration of regulated pathway for calcitonin and constitutive pathway for carcinoembryonic antigen in the same cells of human medullary carcinomas of thyroid glands. Mod Pathol 10(1): 7-11.

22. Busnardo B, Girelli ME, Simioni N, Nacamulli D, Busetto E (1984) Nonparallel patterns of calcitonin and carcinoembryonic antigen levels in the follow-up of medullary thyroid carcinoma. Cancer 53(2): 278-285.

23. Ilantzis C, DeMarte L, Screaton RA, Stanners CP (2002) Deregulated expression of the human tumor marker CEA and CEA family member CEACAM6 disrupts tissue architecture and blocks colonocyte differentiation. Neoplasia New York, NY 4(2): 151-163.

24. Hammarstrom S (1999) The carcinoembryonic antigen (CEA) family: structures, suggested functions and expression in normal and malignant tissues. Seminars in cancer biology 9(2): 67-81.

25. Hashimoto T, Matsubara F, Mizukami Y, Miyazaki I, Michigishi T, et al. (1990) Tumor markers and oncogene expression in thyroid cancer using biochemical and immunohistochemical studies. Endocrinologia japonica 37(2): 247-254.
This work is licensed under Creative Commons Attribution 4.0 License

To Submit Your Article Click Here: Submit Article

DOI: $10.32474 /$ LOJMS.2020.05.000218

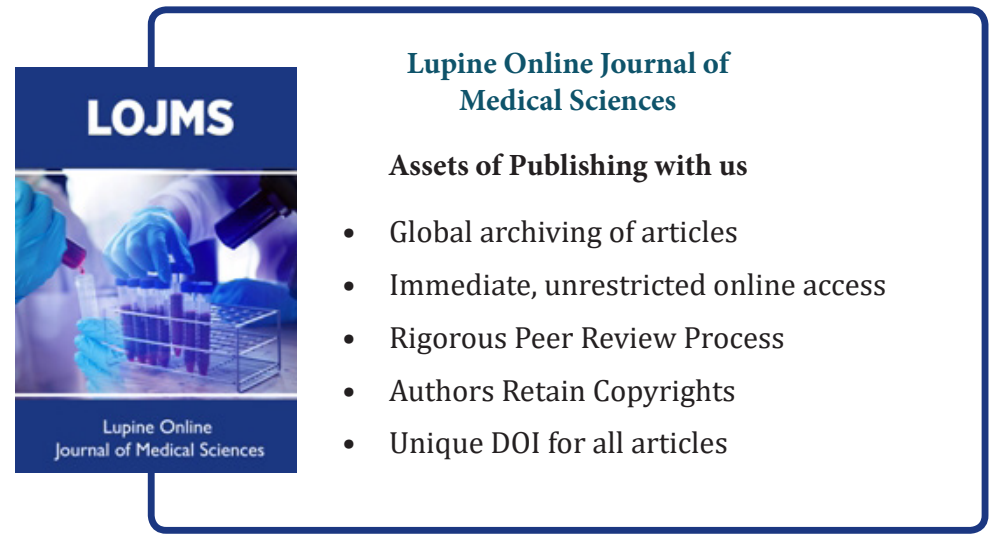

